\title{
La création des LARD
}

Les LARD sont nés d'une toute première association entre le laboratoire de dosimétrie du CEA Fontenay-aux-roses et l'université Paul Sabatier de Toulouse. Elle avait été créée au début des années 60, sous l'impulsion de l'inspecteur général Henri Baïssas (directeur du centre de recherches nucléaires de Fontenayaux-Roses) et de Francis Duhamel (alors Chef du service de radioprotection et de génie radioactif à Saclay) .

Le laboratoire de dosimétrie de Fontenay-aux-roses, alors restreint à une poignée de chercheurs dirigés par Gui Portal, entreprenait des travaux de recherche pour doter la radioprotection de méthodes et de moyens de mesure, alors fort peu développés, pour la dosimétrie des irradiations externes.

Il lui était nécessaire de bénéficier de l'appui d'une structure universitaire capable d'apporter son expérience en matière de recherche et ses connaissances en physique fondamentale. Le professeur Daniel Blanc du Centre de physique atomique (CPA) de l'université Paul Sabatier de Toulouse en fut nommé conseiller scientifique.

Ainsi est née une structure contractuelle qui n'a cessé de se développer, les études de base étant effectuées plus particulièrement à l'université et les études appliquées au CEA.

Le laboratoire de Fontenay, dès le milieu des années 60, se trouva impliqué dans les contrats de recherche de l'Euratome qui lui apportèrent un premier soutien financier et lui permirent d'élargir le cercle de ses études et de se développer jusqu'à créer un service important dont le nom fut plusieurs fois modifié.

Il fut assez rapidement rejoint par celui de Toulouse. Les recherches effectuées en commun en France furent ainsi mises en lumière et de nouveaux moyens financiers leur permirent d'en accélérer encore le développement.

Cependant leurs effectifs respectifs, bien qu'ayant été accrus, ne leur permettaient pas de faire face à tous les besoins de recherche en matière de radioprotection et cela d'autant plus que la collaboration s'étendait progressivement dans le domaine de la radiothérapie.

La nécessité de faire appel à d'autres universités pour renforcer leurs rangs apparut clairement vers la fin des années 70 et une partie des fonds fournis par la

DOI: 10.1051/radiopro:2007011

RADIOPROTECTION - VOL. 41 - @ C EDP Sciences, 2007 
Commission des communautés européennes fut affectée à celles qui souhaitaient se joindre eux dans leurs efforts de recherche.

C'est ainsi que progressivement l'université de Nice (Laboratoire d'émission électronique et de luminescence), celles de Limoges (Laboratoire d'électronique des polymères sous faisceaux ioniques), de Montpellier (université des sciences et techniques du Languedoc), de Saint-Étienne, l'École des Mines de Saint-Étienne (Laboratoire de physique des processus industriels), le Centre de recherches nucléaires de Kronenbourg du CNRS (Service d'application des détecteurs visuels) ainsi que le Laboratoire de biophysique des rayonnements de l'INSERM de Strasbourg les rejoignirent et, pour la plupart, firent leurs premiers pas, à leurs côtés, à la Commission des communautés européennes dont ils venaient de découvrir, grâce à cette collaboration, les vertus et les avantages financiers.

C'est vers 1985 que les laboratoires de Fontenay-aux-Roses et de Toulouse prirent conscience de la nécessité de fédérer tous ces laboratoires dans une structure associative du type 1901, afin de mieux les coordonner et d'apparaître, notamment à la Commission des communautés européennes, comme une entité bien structurée et organisée.

Ils créèrent les LARD et d'autres laboratoires se joignirent à eux par la suite, comme le NPI-DRD de Prague. Ce fut leur force.

Les statuts furent établis en 1985. Le texte constitutif est précédé de la note liminaire suivante : «La radiophysique et la dosimétrie constituent des domaines scientifiques et techniques très diversifiés qui comprennent notamment la radioprotection, certaines applications médicales, l'industrie et la recherche. Ces activités sont développées dans des laboratoires qui restent relativement isolés. Leurs travaux ne sont connus que par leurs publications dans des revues spécialisées et par une communauté scientifique restreinte. L'organisation des LARD facilite la diffusion de l'information concernant ces disciplines, leurs potentialités et les résultats obtenus. Elle assure la coordination entre les chercheurs et incite et facilitera les échanges et les concertations. Les LARD répartissent, après concertation entre les laboratoires intéressés, les travaux demandés en fonction des spécialisations. ».

Les LARD constituaient donc au niveau français un groupe similaire à l'Eurados qui regroupait les laboratoires européens qui effectuaient des recherches en dosimétrie sous le chapeau de la Commission des communautés européennes.

Les laboratoires associés organisèrent chaque année, sous une présidence périodiquement renouvelée, des réunions scientifiques au cours desquelles chacun 
exposait le résultat de ses travaux et présentait ses projets de programmes futurs. Des rapports de synthèse ont été périodiquement publiés.

La coordination de l'ensemble était décidée à cette occasion, ce qui facilita la proposition de programmes à la Commission des communautés européennes.

C'est avec un plaisir que chacun imagine que les deux présidents fondateurs qui sont depuis longtemps à la retraite constatent que, vingt ans après, les LARD sont encore très dynamiques. Les nouveaux responsables des laboratoires ont bien compris la nécessité de l'entraide et de la cohésion en matière de recherche.

Continuez sur votre lancée... Ceux qui vous ont entraînés sur la piste de l'association sont fiers de vous.

Daniel Blanc

Co-fondateur des LARD

Professeur émérite à l'université Paul Sabatier

Directeur du CPAT

Gui Portal

Co-fondateur des LARD

Ancien Chef du service de dosimétrie au CEA/FAR 